\title{
How japanese citizens faced the covid-19 pandemic?: Exploration from twitter
}

\author{
Josimar E. Chire Saire ${ }^{1}$ and Akihiro Masuyama ${ }^{2}$ \\ 1 Institute of Mathematics and Computer Science (ICMC), University of São Paulo (USP), \\ Sao Carlos, SP, Brazil \\ 2 Faculty of Psychology, Iro Sosei University \\ Fukushima, Japan \\ jecs89@usp.br, ak.masuyama@gmail.com
}

\begin{abstract}
Covid-19 outbreak started on December in China, and fastly it was propagated in all the world. Later, during the second week of March, it was declared as pandemic by World Health Organization. Japan has the first case on January 3, 2020 and the first report deaths was on February 18. The scope of this work is to analyze how Japanese people faced the pandemic from January 1 to September 15. The approach for the analysis is based on Text Mining of collected data from Social Network Twitter and Google Trends. The preliminary results are: Twitter can reflect the situation of Japanese citizens during the pandemic and encouraging messages were related to Covid-19 keyword.
\end{abstract}

Keywords: Covid-19 · Text Mining · Twitter Analytics · Google Trends · Coronavirus · Japan

\section{$1 \quad$ Introduction}

The breakout of coronavirus disease (COVID-19) is still continually inflicting hardship on the world. As of early December, according to the report of World Health Organization (WHO), there have been over 65 million confirmed-cases and 1.5 million deaths. Not only medical impact of COVID-19 per se, but social impact have also been huge; self quarantine, locked down, and social distancing. As contrary to past pandemic situation, in 2020, many people around the world were using the Internet, and social networking sites (SNS) were flooded with information about the COVID-19 pandemic. Thus, the pandemic of this time is also referred to as "infodemic" because social media makes information about disease faster and further like a virus [5].

According to Eysenbach [2], current infodemic situation should be managed by following four aspects: 1) information monitoring (infoveillance), 2) building eHealth Literacy and science literacy capacity, 3) encouraging knowledge refinement and quality improvement processes, and 4) accurate and timely knowledge translation. Eysenbach also proposed the "information cake model", which is a model of the propagation of information as a wedding cake. In this model, social media is the largest cake in the bottom row, and while it has a lot of information, the most reliable "Science" in the top row is slow to propagate. Therefore, in the current situation, which is considered to be an infodemic, it is necessary to understand the flow of information by carefully examining what kind of content is posted on social media, which has the largest volume of information.

On Twitter, one of the major social media, several researchers have already developed data set of tweet, reporting that a spike in COVID-19-related Tweets at a time of reports of rising numbers of infections and major political decisions such as the lockdown and international travel restrictions $[1,3]$.

However, the data set of these Tweets consists of only English-speaking countries, and little is known about the characteristics of Tweet in countries where English is not the first language, such as Japan. Unlike Europe and the United States, the number of COVID-19 infections in Japan is relatively low [4], and it is possible that these social media analyses have not been well explored. However, there are a large number of Twitter accounts in Japan (The second leading country of Twitter user; 51.9 million user), and many tweets are expected to exist even in an infodemic situation.

Thus, the aim of this study was to primary investigate the COVID-19 related Tweet in Japan. Specifically, we extracted tweets related to COVID-19 in Japanese tweets from January to September of 2020, and examined which words were strongly associated with it. In this study, we examined tweets from Tokyo, the most populous city in Japan. 


\section{Methodology}

This section is explaining the methodology is used to develop the present study. The actual methodology is inspired in a classical Data Mining approach, and the steps are:

- Selecting scope

- Setting query

- Pre-processing Text

- Visualization

Each step of the methodology is explained in the next subsections. From the selection of the scope to visualization.

\subsection{Selecting scope}

The actual pandemic is a phenomenon which has generated many consequences in different levels in each country, continent and world. The study is focusing in the country of Japan, in the capital named Tokyo.

\subsection{Setting query}

The collection process is through Twitter Search function, with the next parameters:

- date: 01-01-2020 to 15-09-2020,

- terms: Corona

- geolocalization: see Fig. 1

- language: Japanese

- radius: $50 \mathrm{~km}$

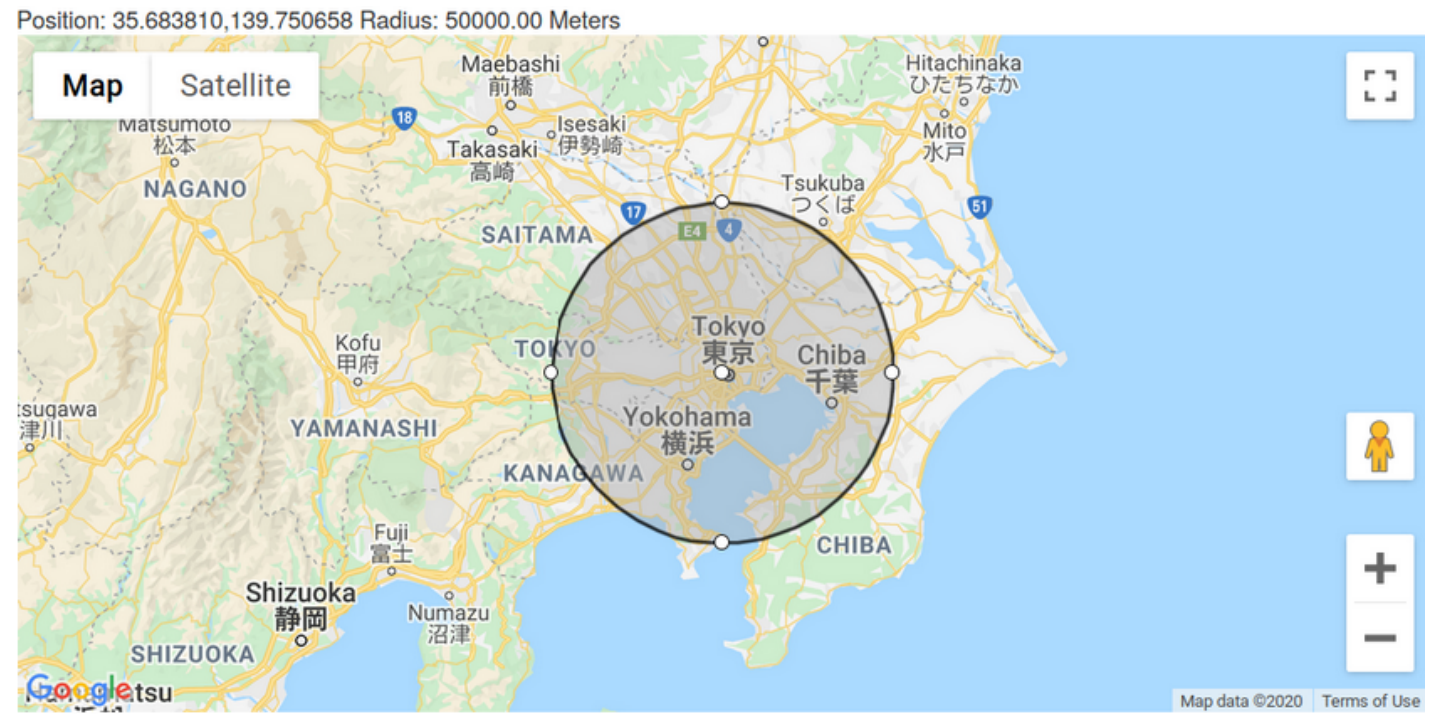

Fig. 1. Tokyo, Japan 


\subsection{Pre-processing Text}

- Clean symbols, characters, i.e. \#, ; and more

- Delete urls/hyperlinks

- Clean stopwords

Stopwords are words with no additional meaning and they are strongly related to the language. Considering, the study is working with Japanese, the proposed terms by Gene Diaz ${ }^{3}$ were used, some of them are: ['asoko','axtu','ano','anokata','anohito','ari','arimasu', 'aru', 'are', 'i', 'iu', 'imasu','iru']

\subsection{Visualization}

- Bar plots to show the frequency of tweets per day, per month

- Cloud of words to visualize the most frequent terms per month

\section{Results and discussion}

This sections is presenting the features of the collected data and the results to answer question to perform the analysis.

\subsection{Dataset Description}

This subsection presents the description of the collected data. Besides, a visualization of the distribution of posts per day is presented in Fig. 2.

- keywords: 'Corona', 'Shingata Corona'

- range data: Jan o1 - Sep 14

- number of collected tweets: 383,053

- number of unique tweets: 318,301

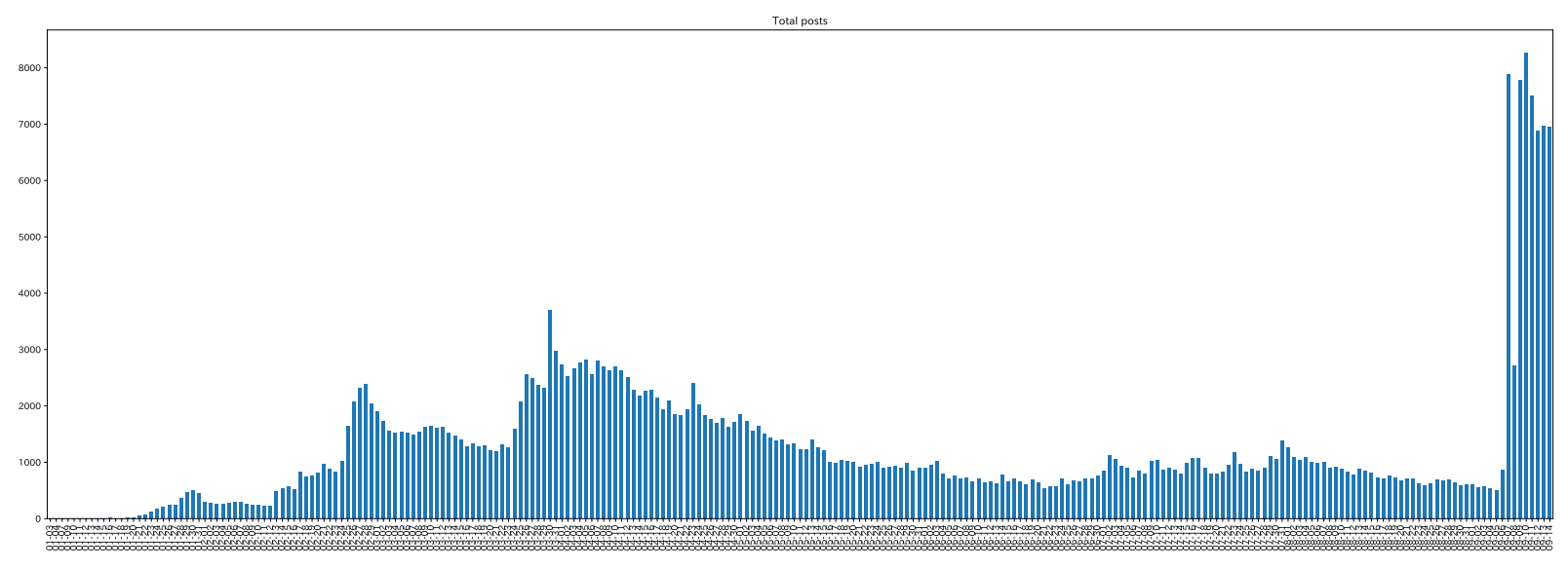

Fig. 2. Frequency per day

\footnotetext{
3 The terms are available in https://github.com/stopwords-iso/stopwords-ja/blob/master/stopwords-ja.txt
} 


\subsection{What were the people talking about?}

This step is purposed to understand what were the most frequent terms during the pandemic, Fig. 3 presents the most used terms during this period.

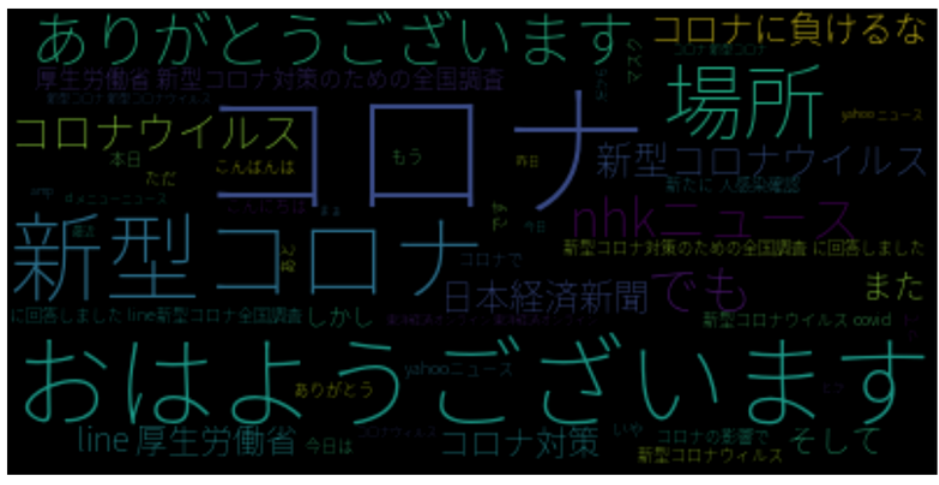

Fig. 3. Most frequent terms

\subsection{What terms were more frequent hashtags during the period?}

Processing the data to get the most frequent hashtags to understand the flow of data between the users. Then, the top 50 during the period of study are presented in Fig. 4.

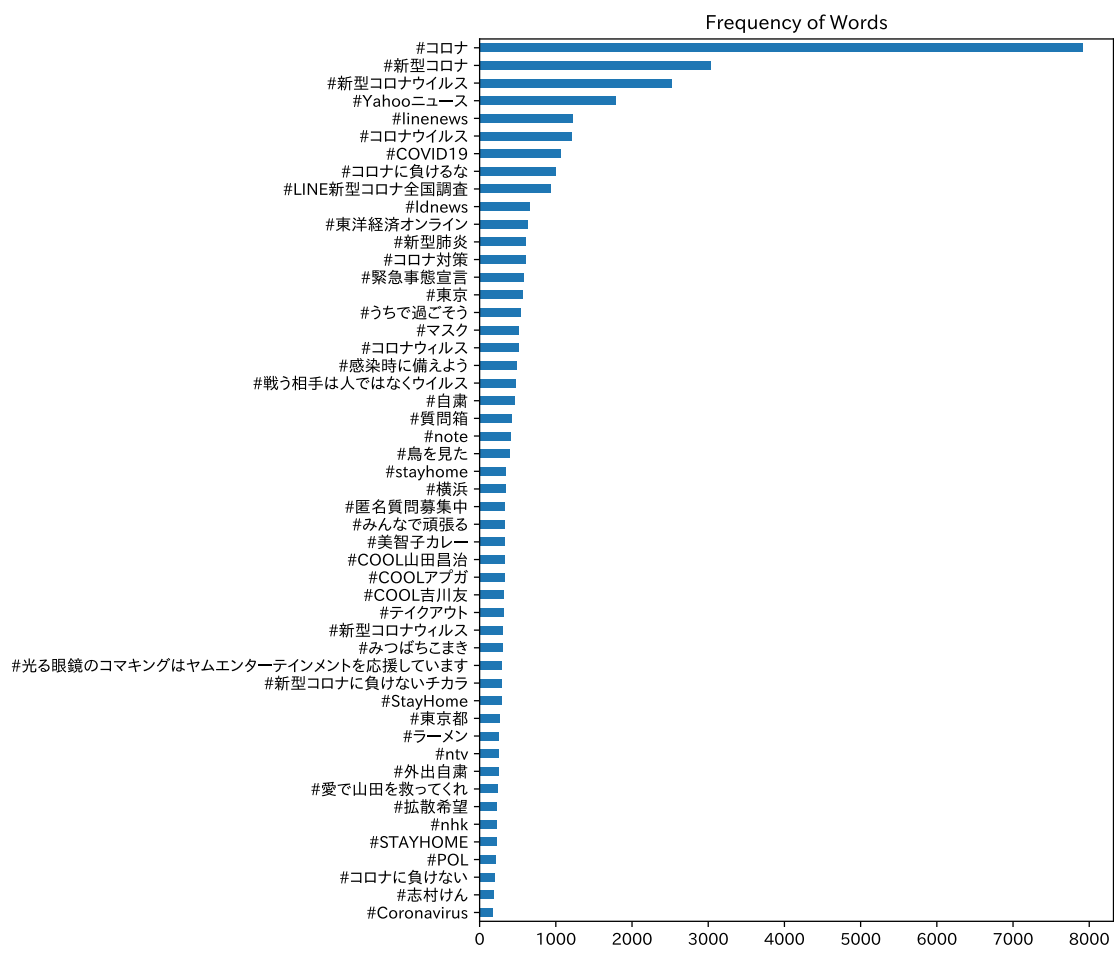

Fig. 4. More frequent hashtags 


\subsection{Can Google trends reflect behaviour of the users?}

Considering the distribution of posts in Twitter, and overlapping is performed to know the relationship between Twitter posts and Google Trends.

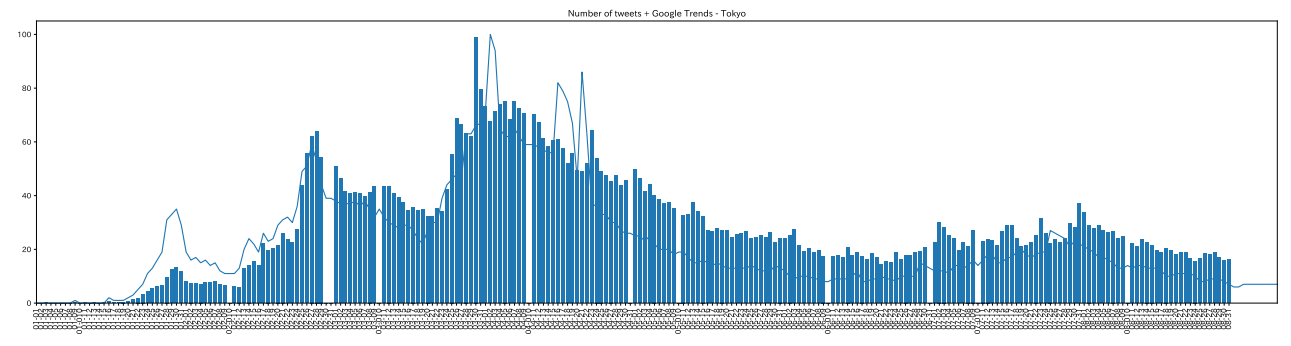

Fig. 5. Twitter posts and Google Trends

Then, it is possible to notice in Fig. 5 there is a relationship between both data sources. And this is related to interest of users on the topic about coronavirus.

\section{Discussion}

First, in the search for COVID-19 related words on Google Trends, the number of searches for the words gradually increased from January, when COVID-19 became a hot topic in Japan, to a very large number of searches from the end of March to April. After that, it maintained a high level in May, but showed a decreasing trend from June.This suggests that the Japanese were interested in unknown viruses and were investigating them. Throughout all months, the most common search word and tweet was "Corona" which may indicate an increase in interest among Japanese.

Next, in the analysis of tweets, "News from Japan Broadcasting Corporation", "Japan Economic Times: Nikkei", and "Ministry of Health, Labour and Welfare" appeared in the word cloud in relation to connection with "Corona" throughout all months. The fact that these highly trusted media in Japan were frequently searched suggests that Japanese people were seeking accurate information. In addition to the news, the "Line of Ministry of Health, Labour and Welfare" was also visualized as a connection with COVID-19 related words. This might be due to the interest in the nationwide survey conducted by the Ministry of Health, Labor and Welfare using the chat app "Line," which is widely used in Japan.

In this survey, demographic data such as age and occupation and the health conditions such as experience of fever, anxiety, and worry were investigated. It is also suggested that there is a growing interest in this type of survey and online surveys at the national level. In the monthly word cloud, "Don't lose to COVID-19" and Let's unite and do our best"are visualized in June and July. This is a word for overcoming a pandemic, suggesting that psychological resilience and encouragement are on the rise. Considering that encouragement related words were not visible before May and that the words related to news appeared from March to May, the following psychological changes can be inferred: the pandemic has begun in January, and then Japanese people were gathering information of COVID-19 from reliable media to understand the situation of COVID-19 and the pandemic. after that, they were trying to overcome the social impact. In fact, words related to "Let's do our best" showed a sustained upward trend until August.

As conclusion, this study was aimed to investigate COVID-19-related tweets from January to September 2020 in Japan. The results showed that tweets in Japan showed a strong interest in information with high reliability. In addition, from June 2020, tweets related to encouragement were also strongly associated with COVID-19 related words.

\section{References}

1. Chen, E., Lerman, K., Ferrara, E.: Tracking social media discourse about the covid-19 pandemic: Development of a public coronavirus twitter data set. JMIR Public Health Surveill 6(2), e19273 (May 2020). https://doi.org/10.2196/19273, http://publichealth.jmir.org/2020/2/e19273/ 
2. Eysenbach, G.: How to fight an infodemic: The four pillars of infodemic management. J Med Internet Res 22(6), e21820 (Jun 2020). https://doi.org/10.2196/21820, http: //www . jmir.org/2020/6/e21820/

3. Rahman, S., Ramakrishnan, T., Ngamassi, L.: Impact of social media use on student satisfaction in higher education. Higher Education Quarterly 74(3), 304-319 (2020). https://doi.org/https://doi.org/10.1111/hequ.12228, https://onlinelibrary.wiley.com/doi/abs/10.1111/hequ.12228

4. WHO: Coronavirus disease (covid-2019) situation reports. Accessed on 27 April, 2021 in url= https://www.who.int/emergencies/diseases/novel-coronavirus-2019/situation-reports (2021)

5. Zarocostas, J.: How to fight an infodemic. The Lancet 395(10225), 676 (2020). https://doi.org/https://doi.org/10.1016/S0140-6736(20)30461-X, https://www.sciencedirect.com/ science/article/pii/S014067362030461X 